\title{
EFECTIVIDAD DE LA LIMPIEZA ULTRASÓNICA DE CONDUCTOS LATERALES SIMULADOS POSTERIOR A LA UTILIZACIÓN DE UN DRESSING DE HIDRÓXIDO DE CALCIO
}

${ }^{1}$ Paola Andrea Gómez M., ${ }^{2}$ Carlos Alberto Ojeda C., ${ }^{3}$ Martha Liliana Rincón $R$.

${ }^{1}$ Odontóloga, Pontificia U. Javeriana, Estudiante de II año de la Especialización en Endodoncia,

U. Santo Tomás, ${ }^{2}$ Odontólogo, U. Nacional de Colombia, Estudiante de II año de la Especialización en Endodoncia,

U. Santo Tomás, ${ }^{3}$ Odontóloga, U. Santo Tomás, Especialista en Endodoncia, U. Santo Tomás, Docente, U. Santo Tomás.

\author{
Autor responsable de la correspondencia: Paola Andrea Gómez M.
}

Correo electrónico: paolagomez18@ hotmail.com

\begin{abstract}
RESUMEN
Objetivo: Comparar la efectividad del hipoclorito de sodio al 5,25\% sólo y activado ultrasónicamente, para remover el dressing de hidróxido de calcio de conductos laterales simulados.

Materiales y métodos: Se realizó un estudio experimental in vitro con 24 dientes humanos uniradiculares recién extraídos, a los que se les realizaron conductos laterales simulados; fueron instrumentados y obturados con un dressing de hidróxido de calcio y almacenados por siete días a 370 $\mathrm{C}$ en $100 \%$ de humedad. Los dientes se asignaron mediante una aleatorización por bloques para ser irrigados con NaOCl al 5,25\% (grupo 0) y con NaOCl al 5,25\% activado ultrasónicamente (grupo 1). Se usó la misma técnica para obturar ambos grupos. Setomó radiografía y se evaluó cada diente, identificando el número de conductos simulados obturados. Para el plan de análisis se consideró aplicar el test de chi2, test de rangos de Wilcoxon o prueba t según la naturaleza y la distribución de las variables.

Resultados: No se encontraron diferencias estadísticamente significativas entre los dos procedimientos.

Conclusiones: El hipoclorito de sodio al 5,25\% sólo o activado con ultrasonido remueve igual el dressing de hidróxido de calcio de conductos laterales simulados de diámetro pequeño. [Gómez PA, Ojeda CA, Rincón ML. Efectividad de la limpieza ultrasónica de conductos laterales simulados posterior a la utilización de un dressing de hidróxido de calcio. Ustasalud Odontología 2006; 5: 9 - 14]
\end{abstract}

Palabras clave: Irrigación ultrasónica, Conductos laterales, Hidróxido de calcio.

\section{EFFECTIVENESS OF ULTRASONIC CLEANING OF SIMULATED LATERAL CANALS FOLLOWING A DRESSING OF CALCIUM HYDROXIDE}

\begin{abstract}
Purpose: To compare the effectiveness of sodium hypochlorite 5,25\% alone and ultrasonically activated on the calcium hydroxide dressing elimination from simulated lateral canals.

Material and methods: An experimental, in vitro study with 24 recently extracted single root human teeth were created simulate lateral canals and instrumented to the w orking length, each tooth was filled with a calcium hydroxide paste and incubated at $37^{\circ} \mathrm{C}, 100 \%$ humidity for seven days. Teeth were randomly divided in to groups, in group 0 the dressing was removed using sodium hypochlorite irrigation and master file, and group 1 the dressing was removed using sodium hypochlorite irrigation and a \# 15 activated $U$ file during one minute. Both groups were obturated using termomechanical compaction. Postoperative radiographs were taken and evaluated; data were analyzed statistically using Mann Whitney or T student and chi square test.

Results: No statistically significant differences were found betw een both groups 0 and 1 .

Conclusions: The sodium hypochlorite $5.25 \%$ alone or activated with ultrasound don't remove completely dressing of hydroxide of calcium of simulated lateral canals of small diameter.
\end{abstract}

Key words: Ultrasonic irrigation, Lateral canals, Calcium hydroxide, Thermomechanical compaction.

Recibido para publicación: 4 deabril de2006. Aceptado para publicación: 6demayo de2006. 


\section{INTRODUCCIÓN}

El hidróxido de calcio se usa como medicamento temporal intraconducto en diferentes situaciones clínicas;, éste debe penetrar en todo el sistema de conductos para que cumpla su función. DeDeus y Horizonteencontraron que un 27\% de 1140 dientes estudiados, tenían evidencia de conductos laterales, ${ }^{2}$ Rubach y Mitchell detectaron conductos laterales en el $45 \%$ de 74 dientes estudiados, la mayoría de ellos en el tercio apical. ${ }^{3}$

La incompleta eliminación del dressing de los conductos laterales de diámetro pequeño y de la porción apical puede ocasionar fallas en la obturación tridimensional del sistema de conductos radiculares y, en algunos casos, el fracaso del tratamiento. ${ }^{4}$

El empleo de sustancias decalcificadoras como el ETDA al $17 \%$ junto con el hipoclorito de sodio al 5,25\% han resultado exitosos para solucionar dicho problema, pero con el inconveniente del debilitamiento de la estructura dentinaria radicular. ${ }^{5}$

Otro elemento utilizado en la limpieza de conductos es el ultrasonido, que puede mejorar la limpieza de los conductos laterales, accesorios y túbulos dentinales por medio de vibraciones ultrasónicas. La energía ultrasónica pasa a través de la solución irrigadora y ejerce su corriente acústica o efecto de restregado sobre las paredes del conducto. ${ }^{6}$ Cameron, en 1987, concluyó que existía una relación de sinergismo entreel hipoclorito de sodio y el ultrasonido durante la irrigación. ${ }^{7}$ Otra variable que influye en la obturación de conductos laterales es la técnica de obturación empleada. Zmener y Gimenes, en 1991, encontraron que la compactación termomecánica proporcionaba un conducto bien obturado con una masa más homogénea de gutapercha. ${ }^{8}$

El propósito de este estudio fue comparar la efectividad del hipoclorito de sodio al 5,25\% sólo y activado ultrasónicamente, para remover el dressing de hidróxido de calcio de conductos laterales simulados

\section{MATERIALES Y MÉTODOS}

Estudio experimental, in vitro, donde setomaron 24 dientes unirradiculares, recientemente extraídos, obtenidos de pacientes atendidos en la consulta de uno de los investigadores. Las variables fueron recolectadas en un formato diseñado para tal fin que incluía además de número de conductos simulados obturados, su ubicación por tercios de los mismos.

Los dientes fueron almacenados en formalina al $10 \%$. Se les retiró la corona clínica por medio de un disco de carburo (№ 1300480 Keystone Ind.) de baja velocidad con refrigeración constante en sentido perpendicular al eje mayor del diente, y se obtuvieron raíces de $14 \mathrm{~mm}$ de longitud.

Los dientes fueron instrumentados manualmente con la técnica Crown-Down por un mismo operador hasta la longitud de trabajo (13.5 mm), verificada visualmente. La instrumentación serealizó hasta una lima apical principal № 40 (K-Flexofile, taper 02, 21mm, Dentsply/ Maillefer); se realizó la irrigación constante por medio de una jeringa con aguja larga de bisel lateral de seguridad, calibre 27 (Monoject/Tyco UK.) con $3 \mathrm{ml}$ de hipoclorito de sodio al 5,25\%, entre cada lima.

En cada uno de los dientes se simularon seis conductos laterales con una fresa LN (calibre 006 Dentsply/Maillefer); tres conductos en la superficie mesial y distal, dos en el tercio apical, dos en el tercio medio y dos en el tercio coronal, de la siguiente forma: se tomó la fresa LN al máximo de velocidad con un micromotor y contra-ángulo de baja velocidad (Kavo/Germany) en dirección perpendicular al eje mayor del diente, en ese momento se permeabilizó el conducto simulado con una lima preserie \# 10.

Cada diente se irrigó al final con $3 \mathrm{ml}$ de hipoclorito de sodio al 5,25\% y $3 \mathrm{ml}$ deEDTA al 17\% (Eufar/Col.), dejándolo por un minuto dentro del conducto, con el fin de eliminar el smear layer y permitir la entrada del dressing; serealizó una nueva irrigación con $3 \mathrm{ml}$ dehipoclorito de sodio al $5,25 \%$ y se seco el conducto con puntas de papel № 40 (Dentsply/Maillefer).

$\mathrm{El} \mathrm{Ca}(\mathrm{OH})_{2}$ (Calcifar de Eufar/Col.), fue llevado al conducto con la jeringa, en sentido ápico-coronal, $1 \mathrm{~mm}$ corto de la longitud de trabajo. La entrada al conducto fue temporalmente sellada con cemento (Coltosol/ Coltene), con un espesor de $3 \mathrm{~mm}$. No se tomaron radiografías de control después de aplicar el hidróxido de calcio.

Los dientes fueron llevados en cajas acrílicas numeradas y almacenados en una incubadora (Memmert / Germany) a $37^{\circ} \mathrm{C}$ durante 7 días en una humedad del

10 
$100 \%$. Posteriormente, se retiró el cemento de sellado temporal con una fresa redonda de diamante de grano medio (SS White Burs, Inc) y una cucharilla metálica afilada.

Los dientes fueron divididos aleatoriamente en dos bloques de 12 dientes cada uno:

Grupo 0: los dientes fueron irrigados con $6 \mathrm{ml}$ de $\mathrm{NaOCl}$ al 5,25\%, previa utilización de la lima apical principal, para facilitar el desalojo del material; los conductos fueron secados con puntas de papel № 40 hasta que se observaron completamente secos.

El cemento a base de resina Topseal (Dentsply/Maillefer) se preparó según las indicaciones del fabricante y se impregnaron las paredes dentinarias con éste por medio del cono principal haciéndolo girar lenta y manualmente dentro del conducto, en el sentido de las manecillas del reloj; se introdujo un espaciador № A 30 (Dentsply/ Maillefer) hasta un milímetro antes de la longitud total de trabajo y se introdujo un cono № 25 impregnado con el cemento sellador, compactado siguiendo la técnica de condensación lateral, hasta que se garantizó la obturación de los $3 \mathrm{~mm}$ apicales.

Posteriormente, fue introducido un instrumento de obturación termomecánica № 40 (25 mm de longitud, Guttacondensor, Dentsply/Maillefer) en movimiento, a 20.000 r.p.m. aproximadamente hasta una longitud de $3 \mathrm{~mm}$ antes de la longitud de trabajo, durante 10 segundos y retirado del conducto estando aún en movimiento. La gutapercha fue compactada en sentido vertical con un instrumento № 3/2 (Dentsply/Maillefer).

Grupo 1: dientes irrigados con $3 \mathrm{ml}$ de $\mathrm{NaOCl} 5.25 \%$ con ayuda de la lima apical maestra para facilitar el retiro del hidróxido de calcio. Se introdujo una lima ultrasónica № 15 (U File № 15, de $33 \mathrm{~mm}$ de longitud Varios/ NSK) hasta $3 \mathrm{~mm}$ antes de la longitud de trabajo, activada por una unidad de ultrasonido (Varios 550/NSK, Inc.) en poder 3 y velocidad $\mathrm{E}$ (medio) durante 60 segundos. El conducto fue irrigado con $3 \mathrm{ml}$ más de hipoclorito de sodio al 5,25\% y secado con puntas de papel № 40 (Taper 02, Dentsply/Maillefer Suiza), hasta que se observaron completamente secas. La técnica de aplicación de cemento sellador y de obturación fue igual a la utilizada en el grupo 0.
Se tomaron radiografías de cada uno de los dientes, con incidencia desde la superficie vestibular, se revelaron, se fijaron y se guardaron en sobres separados y codificados. Posteriormente, un evaluador que desconocía el procedimiento al que había sido sometido cada uno de los dientes, identificó el número de conductos laterales simulados completamente obturados con su ubicación.

El plan de análisis se realizó de la siguiente manera: se describieron cada una de las variables mediante medidas de resumen según la naturaleza de las variables y posteriormente, se estableció la relación de conductos obturados según procedimiento mediante t de Student o Test de Rangos deWilcoxon según la distribución de las variables. Adicionalmente, se compararon las proporciones deconductos obturados y no obturados según procedimiento en los tres tercios, mediante el test de Chi Cuadrado.

\section{RESULTADOS}

Al comparar la efectividad del hipoclorito de sodio al $5,25 \%$ sólo y activado ultrasónicamente, para remover el dressing de hidróxido de calcio de conductos laterales simulados, no seencontraron diferencias estadísticamente significativas entre los dos grupos (grupos 0 y 1) (Figu-

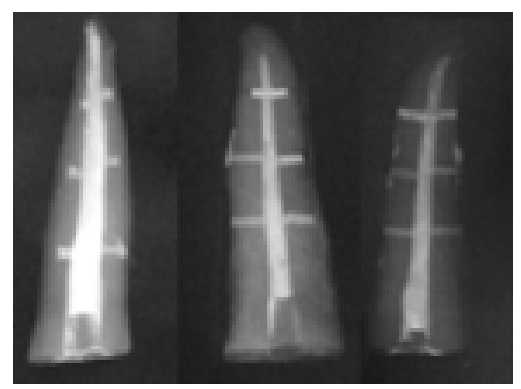

Figura 1. Grupo 0: radiografías de dientes en los cuales el dressing de hidróxido de calcio fue eliminado de los conductos laterales simulados con hipoclorito de sodio al 5,25\%.

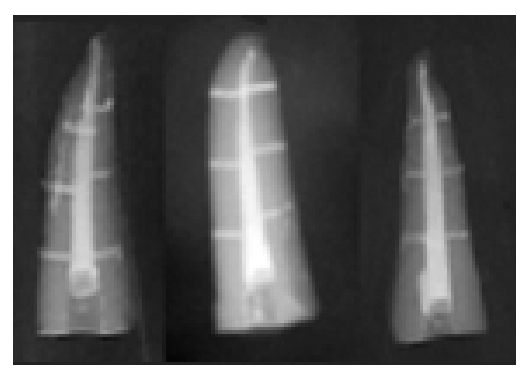

Figura 2. Grupo 1: radiografías de dientes en los cuales el dressing de hidróxido de calcio fue eliminado de los conductos laterales simulados con hipoclorito de sodio activado con ultrasonido. 
Tabla 1. Descripción del promedio total de conductos laterales simulados obturados según el procedimiento.

\begin{tabular}{lcccc}
\hline \multicolumn{1}{c}{ Variable } & Global & Grupo 0 & Grupo 1 & P* \\
\hline $\begin{array}{l}\text { Conductos laterales simulados } \\
\text { obturados }\end{array}$ & 4,125 & 4 & 4,25 & 0,7149 \\
$\begin{array}{l}\text { Conductos laterales simulados no } \\
\text { obturados }\end{array}$ & 1,875 & 2 & 1,75 & 0,7149 \\
\hline
\end{tabular}

$\alpha=0.05 \quad$ "t student

Tabla 2. Descripción del promedio de conductos laterales simulodos obturodos por tercios según procedimienta.

\begin{tabular}{lcccc}
\hline \multicolumn{1}{c}{ Variable } & Global & Grupo 0 & Grupo 1 & P* $^{*}$ \\
\hline Conductos obturados en el tercio coronal & 1,3333 & 1,25 & 1,4166 & 0,6030 \\
Conductos obturodos en el tercio Medio & 1,5416 & 1,5 & 1,5833 & $0,7640^{* *}$ \\
Conductos obturodos en el tercio Apical & 1,25 & 1,25 & 1,25 & 1,0 \\
\hline
\end{tabular}

$a=0.05 \quad$ "t student $\quad$ "* Mann-Whitney

Tabla 3. Comparación de la proporción de conductos laterales simulados obturados y no obturados por tercios según procedimiento.

\begin{tabular}{|c|c|c|c|c|c|}
\hline \multirow{2}{*}{ LOCALZACIÓN } & \multicolumn{2}{|c|}{ Grupo 0} & \multicolumn{2}{|c|}{ Grupo 1} & \\
\hline & Obturados & No Obturados & Obturados & No Obturados & \\
\hline Tercio Coronal & $\begin{array}{c}15 \\
\text { (62.5\%) }\end{array}$ & $\begin{array}{c}9 \\
(37.596)\end{array}$ & $\begin{array}{c}17 \\
{[708 \%)}\end{array}$ & $\begin{array}{c}7 \\
(29.296)\end{array}$ & $0.5 \% 0$ \\
\hline Tercio Medio & $\begin{array}{c}18 \\
{[75 \% 6]}\end{array}$ & $\begin{array}{c}6 \\
(2590)\end{array}$ & $\begin{array}{c}19 \\
{[79.2 \%)}\end{array}$ & $\begin{array}{c}5 \\
(20.8 \%)\end{array}$ & 0.731 \\
\hline Tercio Apical & $\begin{array}{c}15 \\
\text { (62 5\%) }\end{array}$ & $\begin{array}{c}9 \\
(37.5 \% 6)\end{array}$ & $\begin{array}{c}15 \\
{[62.5 \%]}\end{array}$ & $\begin{array}{c}9 \\
(37.5 \% 6)\end{array}$ & 1.0 \\
\hline Total & $\begin{array}{c}48 \\
(66.796)\end{array}$ & $\begin{array}{c}24 \\
(33.3 \% 6)\end{array}$ & $\begin{array}{c}51 \\
{[70.8 \%]}\end{array}$ & $\begin{array}{c}21 \\
(29.296)\end{array}$ & 0.590 \\
\hline
\end{tabular}

ra 1 y 2). Sin embargo, se observó un promedio ligeramentesuperior de efectividad en la eliminación del dressing con hipoclorito de sodio activado con ultrasonido y una relación 2:1 de conductos obturados con relación a los no obturados sin importar el procedimiento (Tabla 1).

En el análisis estadístico no se observaron diferencias significativas cuando se compararon cada uno de los tercios en ambos grupos y al observar los promedios y proporciones obtenidos en los diferentes tercios, en el mismo grupo; se encontró un ligero aumento en el promedio de conductos laterales simulados obturados en el tercio medio, seguido del tercio coronal y apical (Tabla 2 y 3).

\section{DISCUSIÓN}

Diferentes técnicas han sido utilizadas para eliminar 0 remover el dressing de hidróxido de calcio con el fin de lograr el sellado tridimensional del sistema de conductos con cemento sellador y/o gutapercha, al obtener resultados controversiales. ${ }^{9-16}$

Porkaew y colaboradores, Calt y Server, y Margelos y colaboradores demostraron que la ampliación del conducto con la lima apical principal eirrigación con hipoclorito de sodio no removía completamente el dressing de hidróxido de calcio de las paredes del conducto radicular. $9,13,16$

12 
Goldberg y colaboradores, en el 2002, reportaron la obturación de conductos laterales simulados en un $21.7 \%$ con la misma técnica usada por los autores anteriores; ${ }^{12}$ en este estudio, se encontró, en el grupo donde se utilizó hipoclorito de sodio al 5,25\% y la lima apical principal, un $66.7 \%$ de conductos laterales simulados obturados

Ahmad y colaboradores, en 1987, demostraron que la corriente acústica generada por la irrigación activada con ultrasonido genera grandes fuerzas, suficientes para desalojar detritos en conductos instrumentados. ${ }^{6}$ Lee y colaboradores, en el 2004, compararon la efectividad de la irrigación ultrasónica y la irrigación con jeringa con el fin de remover detritos de irregularidades simuladas en conductos radiculares instrumentados; la irrigación ultrasónica fue capaz de remover mayor cantidad de detritos artificiales en conductos rectos y amplios. ${ }^{17}$

En esta investigación se utilizó el hipoclorito de sodio activado con ultrasonido con el fin de retirar el dressing de hidróxido de calcio de los conductos laterales simuladosy sólo seencontró un ligero aumento en la proporción de conductos obturados, al compararlo con el grupo control (70.8\% y $66.7 \%$, respectivamente), aunque no fue estadísticamente significativo. Sin embargo, al compararlo con estudios anteriores, la limpieza de los conductos, aunque no fue completa, fuesignificativamentemayor.

Goldberg y colaboradores no encontraron diferencias estadísticamente significativas entre los diferentes tercios en el mismo grupo experimental, al evaluar la obturación de conductos laterales simulados posterior a la utilización de un dressing de hidróxido de calcio, ${ }^{12}$ esto coincide con los resultados obtenidos en este estudio.

En esta investigación, se obturaron en el grupo de hipoclorito sólo con lima apical principal un $62.5 \%$ en el tercio coronal, $75 \%$ en el tercio medio y $62.5 \%$ en el tercio apical, lo cual está en desacuerdo con lo encontrado en el anterior estudio, donde obturaron un $15 \%$ en el tercio coronal, $20 \%$ en el tercio medio y $30 \%$ en el tercio apical. Esta diferencia pudo ser el resultado de la diferencia en las técnicas de obturación empleadas.

Aunque se encontró un alto porcentaje de obturación de conductos laterales, los resultados expuestos concuerdan con la mayoría de estudios, que sugieren que el dressing de hidróxido de calcio no es removido completamente de las paredes del conducto con hipoclorito de sodio y limado.

\section{CONCLUSIONES}

1. El hipoclorito de sodio al 5,25\% sólo o activado con ultrasonido no remueve completamente el dressing de hidróxido de calcio en conductos laterales simulados.

2. No hay diferencias en la eliminación del dressing de hidróxido de calcio por tercios radiculares, independiente del procedimiento utilizado.

3. Al utilizar el hipoclorito de sodio al 5,25\% activado con ultrasonido hay un ligero aumento en el número de conductos laterales simulados obturados

4. Sin importar el procedimiento utilizado, se da un ligero aumento en el número de conductos laterales simulados obturados en el tercio medio radicular

\section{Agradecimientos.}

Los autores quieren agradecer a la Doctora Sonia Constaza Concha Sánchez por su orientación y asesoría en el análisis estadístico.

\section{BIBLIOGRAFÍA}

1. Byström A, Sjögren U, Sundqvist G. Healing of periapical lesions of pulpless teeth after endodontic treatment with controlled asepsis. Endod Dent Traumatol 1987; 3: 58 - 63.

2. De Deus QD, Horizonte B. Frequency, location and direction of lateral, secondary, and accessory canals. J Endod 1975; 1: 361 - 366.

3. Rubach WC, Mitchell DF. Periodontal disease, accessory canals and pulp pathosis. J Periodontol 1965; 36: 34 - 38.

4. Nicholls E. Lateral radicular disease due to lateral branching of the root canals. Oral Med Oral Pathol 1963; 16: 839 - 845.

5. Dogan $\mathrm{H}$, Calt S. Effects of chelating agents and sodium hypochlorite on mineral content of root dentin. J Endod 2001; 27: 578 - 580.

6. Ahmad M, Pitt Ford TR Crum LA. Ultrasonic debridement of root canals: an insight into the mechanisms involved. J Endod 1984; 13: $490-499$.

7. Cameron JA. Thesynergistic relationship between ultrasound and sodium hypochlorite: a scanning electron microscope evaluation. J Endod 1987; 13: 541 - 545

8. Zmener 0, Gimenes J. Thermomechanical compaction of guttapercha: a scanning electron microscopestudy. Endod Dent Traumatol 1991; 7: 153 - 157.

9. Calt S, Serper A. Dentinal tubule penetration of root canal sealers after root canal dressing with calcium hydroxide. J Endod 1999; 25: 431 - 433. 
10. Holland R, Alexandre AC, Murata SS, Dos Santos CA. Apical leakagefollowing root canal dressing with calcium hydroxide. Endod Dent Traumatol 1995; 11: 261 - 263.

11. Ricucci D, Langeland K. Incomplete calcium hydroxide removal from the root canal: a case report. Int Endod J 1997; 30: 418 - 421.

12. Goldberg F, Artaza LP. Influence of calcium hydroxide dressing on theobturation of simulated lateral canals.J Endod 2002; 28: 99-101.

13. Margelos J, Eliades G, Verdelis C. Interaction of calcium hydroxide with zinc oxideeugenol type sealers. A potential clinical problem. J Endod 1997; 23: 43 - 48.

14. Lambrianidis T, Margelos J, Beltes P. Removal efficiency of calcium hydroxide dressing from the root canal. J Endod 1999; $25: 85$ - 88.

15. Kim SK, Kim YO. Influence of calcium hydroxide intracanal medication on apical seal. Int Endod J 2002; 35: 623 - 628.

16. Porkaew P, Retief EH, Barfield RD. Effects of calcium hydroxide past as an intracanal medicament on apical seal. J Endod 1990; 16: $369-375$.

17. Lee S-J, Wu M-K, Wesselink PR. The effectiveness of syringe irrigation and ultrasonic to remove debris from simulated irregularities within prepared root canal walls. Int Endod J 2004; 37: $672-678$.

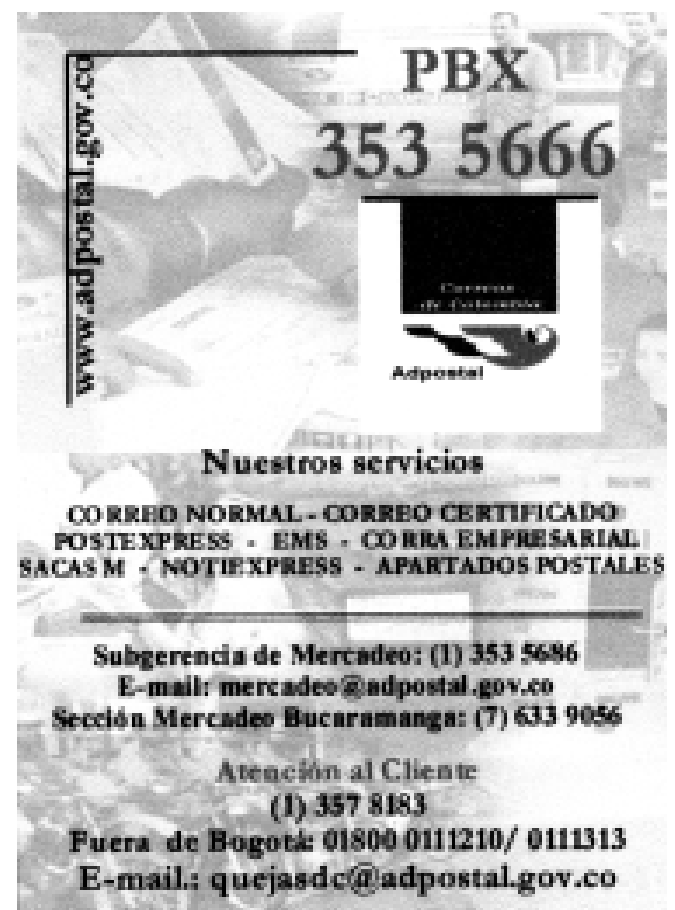

14 